\title{
Systematic review and meta-analysis of the CT imaging characteristics of infectious pneumonia
}

\author{
Yali Liu ${ }^{1,2}$, Xiaohuan Zhou ${ }^{1}$, Xiao Liu ${ }^{1,2}$, Yingjie $\mathbf{Y i}^{3}$, Shanshan Zhang ${ }^{1,2}$, Jihong Wang ${ }^{1}$, Fengjuan Yuan ${ }^{1,2}$ \\ ${ }^{1}$ Pingdingshan University Medical College, Pingdingshan, China; ${ }^{2}$ Philippine Women's University School of Nursing, Manila, Philippines; \\ ${ }^{3}$ Department of Book Cataloguing, Nanyang Medical College, Nanyang, China \\ Contributions: (I) Conception and design: Y Liu, F Yuan; (II) Administrative support: X Zhou, X Liu; (III) Provision of study materials or patients: Y \\ Liu, X Zhou, X Liu, Y Yi, S Zhang; (IV) Collection and assembly of data: All authors; (V) Data analysis and interpretation: Y Yi, S Zhang, J Wang, F \\ Yuan; (VI) Manuscript writing: All authors; (VII) Final approval of manuscript: All authors. \\ Correspondence to: Fengjuan Yuan. Pingdingshan University Medical College, Chongwen Road, Xinhua District, Pingdingshan, China. \\ Email: yfj860901@163.com.
}

\begin{abstract}
Background Since December 2019, there have been cases of infectious pneumonia of unknown cause in Wuhan, Hubei Province, China. On January 12, 2020, the World Health Organization (WHO) named it COVID-19. There are few studies on the clinical characteristics of patients with COVID-19, and results vary widely in sample sizes.

Methods: Chinese and English databases were searched with "Infectious pneumonia", "COVID-19", "CT", "SARS-COV-2", and "Diagnose" as keywords. Rev Man 5.3 software provided by the Cochrane system was used to assess the quality of the included literature.

Results: Of the 18 included studies, ground-glass shadow was the most common computed tomography (CT) sign [95\% confidence interval (CI): 0.79-0.97], followed by thickening of the blood vessels (95\% CI: 0.63-0.78), and pleural thickening (95\% CI: 0.02-0.15). Of the 18 studies, 12 reported that the lesions were externally subpleural (95\% CI: 0.132-0.173), and 6 reported that the lesions were distributed in a single lobe (95\% CI: 0.598-0.841). The heterogeneity test results showed that the morphology of the lesions was cordlike (95\% CI: 0.092-0.172), grid-like (95\% CI: 0.152-0.193), patchy (95\% CI: 0.192-0.313), and nodular (95\% CI: 0.591-0.745).
\end{abstract}

Discussion: Ground-glass opacity was a typical CT manifestation for patients with infectious pneumonia, and CT signs were instrumental in diagnosing this disease.

Keywords: Computed tomography (CT); infectious pneumonia; imaging

Submitted Jul 12, 2021. Accepted for publication Sep 18, 2021.

doi: 10.21037/apm-21-2101

View this article at: https://dx.doi.org/10.21037/apm-21-2101

\section{Introduction}

Pneumonia refers to inflammation in the terminal airways, alveoli, and interstitium of the lungs (1). Pathogenic microorganisms such as bacteria, viruses, fungi and parasites, and physical and chemical factors such as radiation and inhaled foreign bodies may all lead to pneumonia (2). Clinically, it manifests as fever, cough, expectoration, blood in sputum, chest pain, or difficulty breathing. Commonly, pneumonia is not infectious, whereas pneumonia caused by a virus is infectious, such as severe acute respiratory syndrome (SARS), anthrax, and pneumonic plague (3). These different types of pneumonia have different pathogenic mechanisms, causes, and treatment methods.

At present, the COVID-19 pandemic is still spreading around the world. Statistics from the World Health Organization (WHO) reveal that as of January 15, 2020, there were a total of 150 million confirmed cases worldwide and a total of 3.2 million deaths (4). When it comes to a new infectious disease, early detection and early isolation 
is a prerequisite for early treatment (5). A positive virus nucleic acid test result is the gold standard for diagnosing COVID-19. However, as the number of cases gradually increases, asymptomatic infections have appeared. To prevent asymptomatic infections from becoming the source of infection, computed tomography (CT) examination is performed for early diagnosis (6).

CT involves tomographic scanning around a certain part of the human body using $\mathrm{X}$-rays, $\gamma$-rays together with a highly sensitive detector. It is characterized by short scanning time and high resolution (7). CT findings of patients with infectious pneumonia were as follows. Eighty percent of the disease in both lungs was visible; most of them were located in the next lateral band of the pleura in the middle and lower fields of both lungs, but the pleura was often transparent band, less involved in the leaf or intersegmental fissure, not in a clear leaf or segment distribution; the early CT findings were characteristic: observed from the lung window, the pulmonary vascular texture was significantly enhanced, and generally exudate was found in the lobule area, mainly interstitial exudate, so the density was light and often showed ground glass density (8). Nowadays, CT imaging has been widely used in clinical diagnosis to determine disease conditions, and is indispensable for diagnosing infectious pneumonia (9).

Despite a large number of CT-imaging reports on COVID-19 worldwide, they have distinct research results and limited samples (10). In China, an evidencebased foundation to evaluate the CT images of patients with COVID-19 is lacking. The innovation of this study lies in the meta-analysis, which is mainly based on the literature on CT imaging features of infectious pneumonia published since the establishment of the database, and it comprehensively analyzes the imaging features of infectious pneumonia, so as to provide a reliable reference basis for further guiding the clinical treatment of infectious pneumonia. We present the following article in accordance with the PRISMA reporting checklist (available at https:// dx.doi.org/10.21037/apm-21-2101).

\section{Methods}

\section{Literature retrieval}

Relevant literature from CMCI, MEDLINE, EMbase, PubMed, CNKI, China Biomedical Resources Database, Wanfang Database, VIP Database, Baidu Academic Database were retrieved from the establishment date of the database to February 21th, 2020. Relevant studies were analyzed with the Boolean logic retrieval method. The Chinese and English databases were used for a combination search using "Infectious pneumonia", "COVID-19", "CT", "SARS-COV-2", and "Diagnose".

A combined search strategy of free text and subject headings was used for every database. Once confirmed, using a search engine, references were tracked, and after contacting experts and researchers, the latest research progress was acquired.

\section{Literature inclusion and exclusion criteria}

Inclusion criteria: (I) studies on infectious pneumonia; (II) randomized controlled trials (RCTs); (III) compared with clinical anatomy and pathology, the index has high reliability in $95 \%$ confidence interval (CI); (IV) the experimental group underwent CT examination; (V) the diagnosis of infectious pneumonia met the standards of the American Society of Infectious Diseases and the American Thoracic Society.

Exclusion criteria: (I) the research object was special population, restricted by age and gender; (II) studies that were replicated in different journals; (III) literature reviews, abstracts, case reports, or animal experiments; (IV) studies with unavailable complete data; (V) research literature on noninfectious pneumonia patients and animals.

Two senior experts screened the topic, abstract and full text, and if there is any disagreement, they can obtain a consistent conclusion through discussion.

\section{Observation indicators}

CT imaging for patients with infectious pneumonia was evaluated based on lesion positioning, lesion morphology, lesion density, and accompanying signs.

\section{Data extraction}

With a unified Microsoft Excel table, two experts independently extracted the relevant data. Disagreements between experts could be resolved through discussion and consensus, or a third expert could be asked to arbitrate. The extracted data were as follows: (I) research title; (II) published first author and years; (III) study design type; (IV) general information of subjects: gender, age, sample size, country; (V) extraction evaluation index: CT image features. 
Table 1 Evaluation items proposed by the NICE

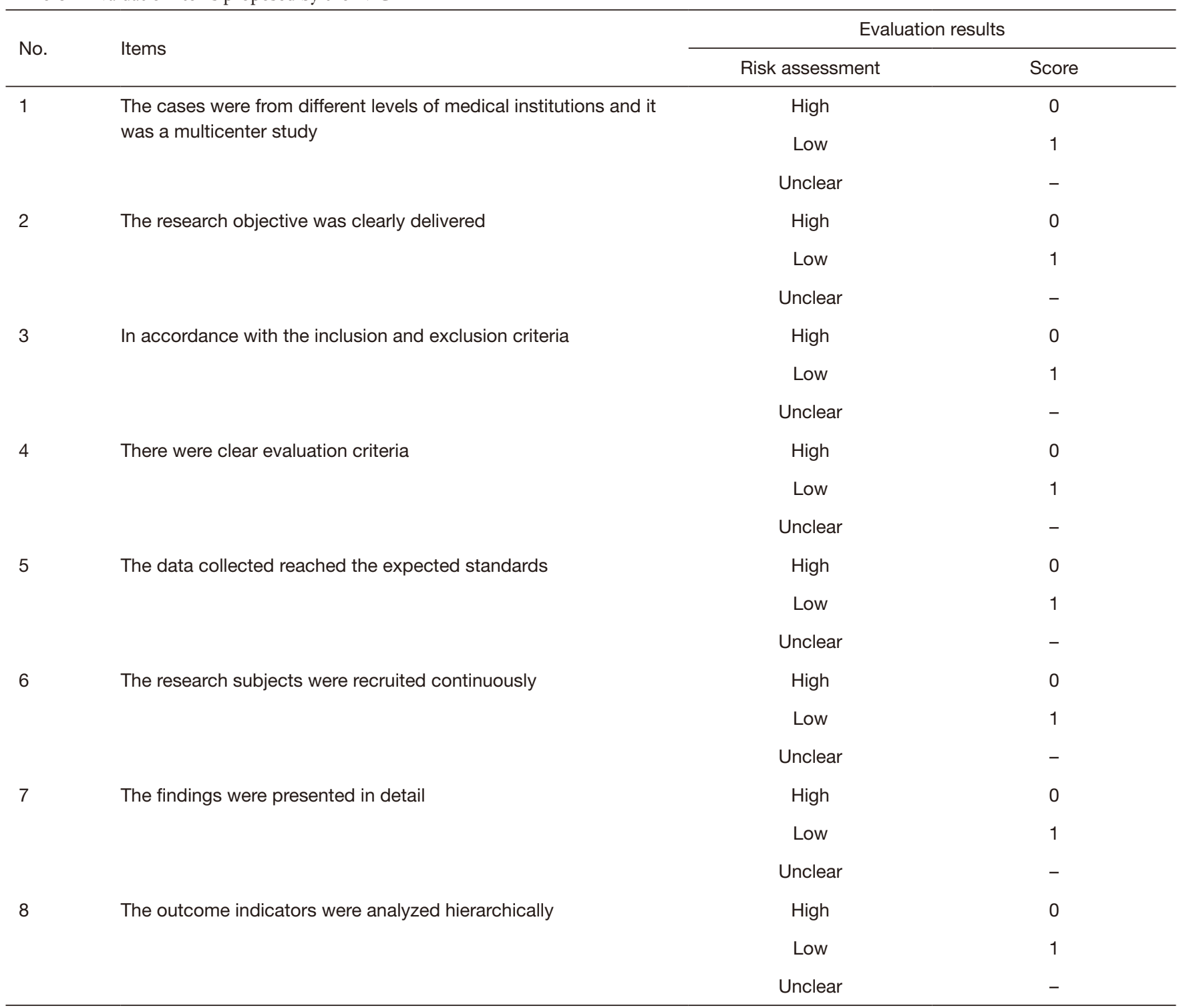

NICE, National Institute of Clinical Excellence.

\section{Risk of bias assessment and quality evaluation}

The risk of bias and quality assessment should be carried out by two researchers at the same time. If the two researchers were disagreed, the results would be determined through discussion. As for quality evaluation, the items proposed by the National Institute of Clinical Excellence (NICE) were adopted, as shown in Table 1. Each item was 1 point, and the total score ranged from $0-8$. The score of each item was 1 point, and the score range was $0-8$ points: $6-8$ points were defined as high-quality research, 3-6 points were defined as medium-quality research, and 0-2 points were defined as low-quality research. Two experts independently carried out quality assessment, and when the opinions were inconsistent, they could obtain consistent conclusions through discussion.

\section{Statistical analysis}

Stata SE 12.0 software (College Station, USA) was used for statistical analysis. The Rev Man 5.3 software provided by Cochrane collaboration network is used to draw the biased risk assessment chart, evaluate the risk bias included in the 


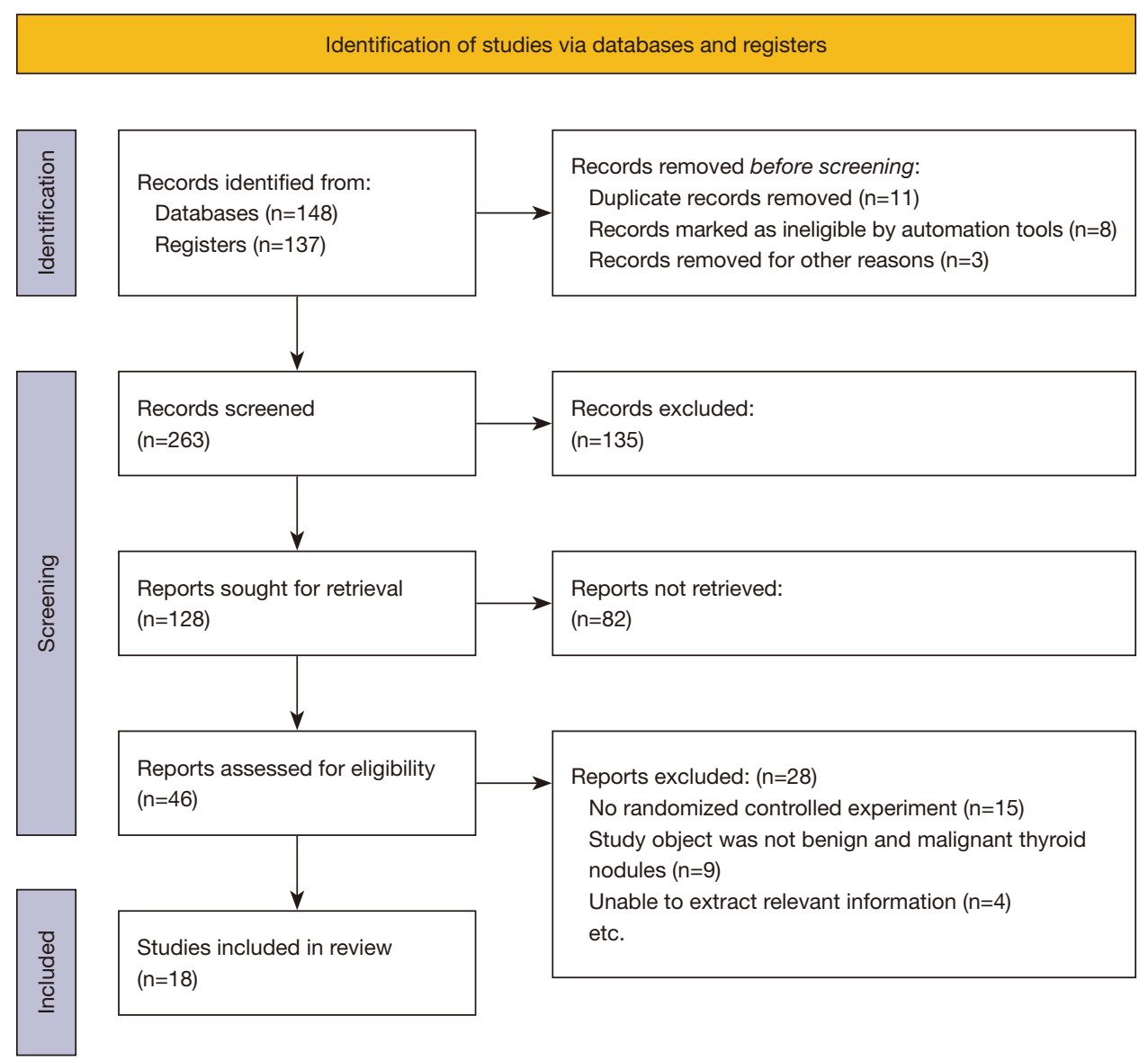

Figure 1 Literature search flowchart.

reference documents, and input the sorted and screened data into the software to draw the corresponding chart. The heterogeneity of each study was explored by $\mathrm{I}^{2}$ test. When $\mathrm{P}>0.01$ and $\mathrm{I}^{2}<50 \%$, the fixed effect model was used for meta-analysis, which indicated that the heterogeneity included in the study was small. When $\mathrm{P}<0.01$ and $\mathrm{I}^{2}>50 \%$, the random effect model was used for meta-analysis, which indicated that the included research was heterogeneous.

\section{Results}

\section{The basic information of the included literature}

One hundred and forty-eight articles were included in this study through the database and 137 through the registry. Eleven literatures published repeatedly were excluded, 8 literatures unqualified were excluded, and 3 literatures for other reasons were excluded, leaving 263 literatures remaining. Read the full text and eliminate 135 articles, leaving 128 articles. Eighty-two cases reports, reviews and conferences were excluded, and 46 qualified articles remained. Fifteen literatures that were not included in the randomized controlled analysis, 9 literatures that were not included in the study of benign and malignant thyroid nodules, and 4 literatures whose relevant information could not be extracted were excluded. Finally, 18 literatures were obtained and entered into the meta-analysis (Figure 1).

Figure 2 shows the results of quality classification. Seven studies scored 6-8 (38.89\%), 10 studies scored 3-6 (55.56\%), and 1 scored between 0-2 (5.55\%).

Eighteen studies met the inclusion criteria, involving a total of 1,586 cases. Of the 18 studies, there were 11 with a small sample size ranging from 35-154. The research subjects were all above 20 years old, as shown in Table 2.

\section{Risk of bias evaluation}

Figures 3,4 show that of the 18 included RCTs, 5 had the 


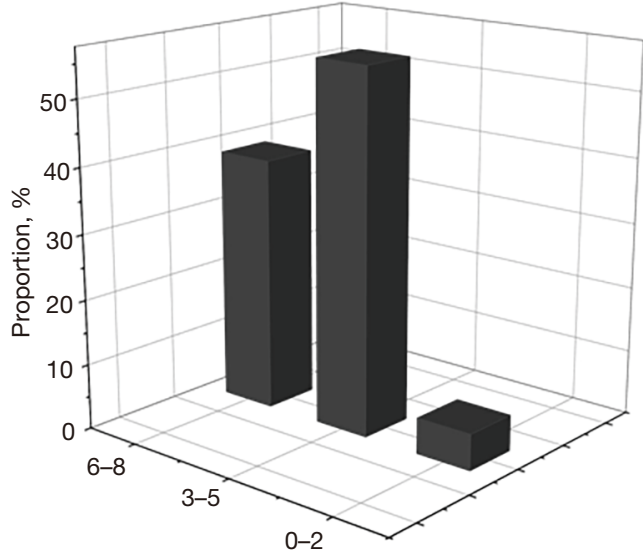

Figure 2 Literature quality classification results. correct random allocation method (11-15), accounting for $27.78 \%$, and only $2(16,17)$ had detailed allocation concealment, accounting for $16.67 \%$. One study (18) was evaluated using the blinding method, accounting for $5.55 \%$, which was not adopted in the other studies.

\section{CT signs}

Figure 5 shows the forest plot of the CT signs of infectious pneumonia. Of the 18 studies, the heterogeneity test results showed that among the CT signs, lung groundglass shadow, lung consolidation shadow, and lung groundglass shadow combined with consolidation exhibited high heterogeneity, with $\mathrm{I}^{2}=96.4 \%>50 \%$. Ground-glass shadow

Table 2 General information of the included studies

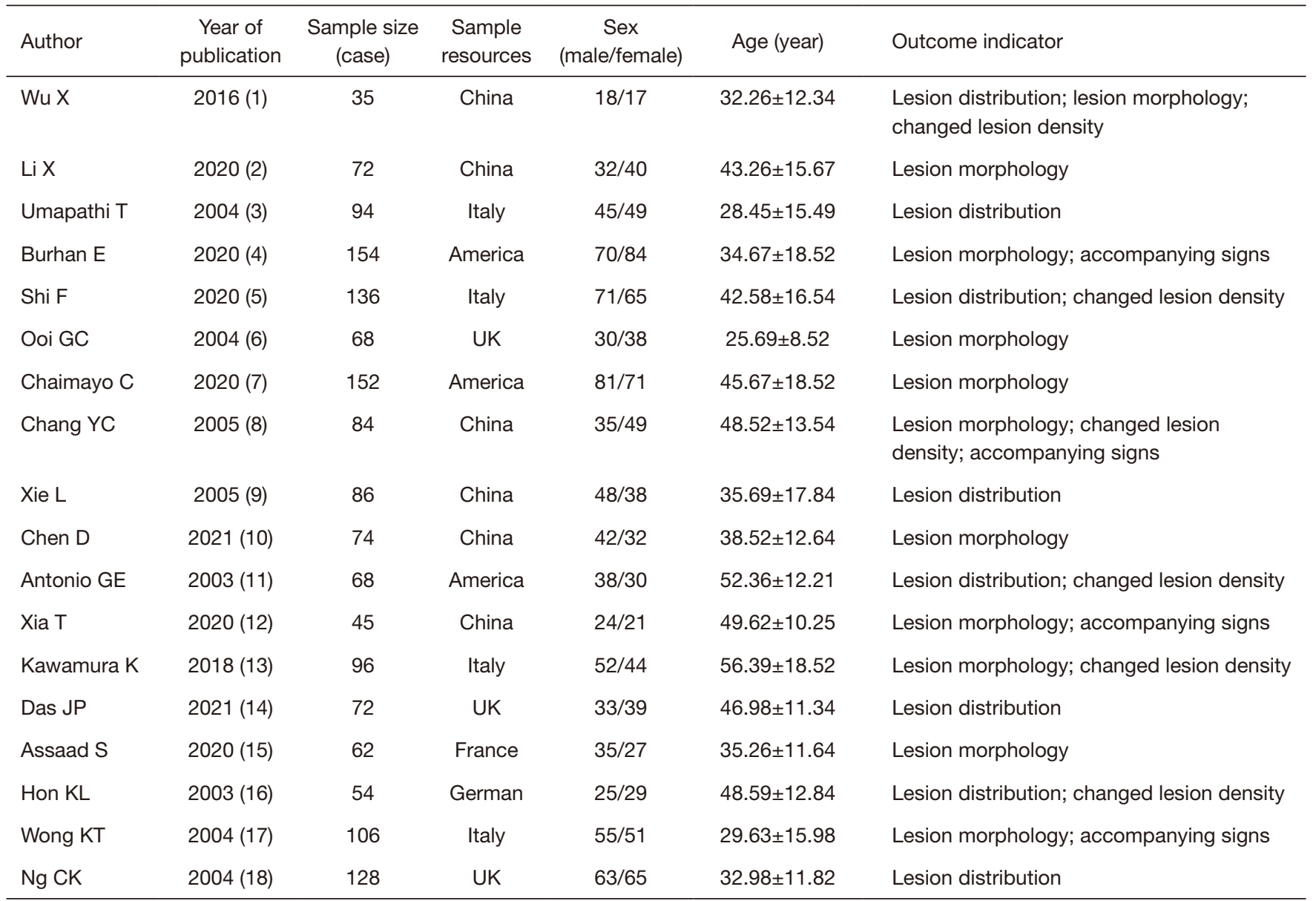




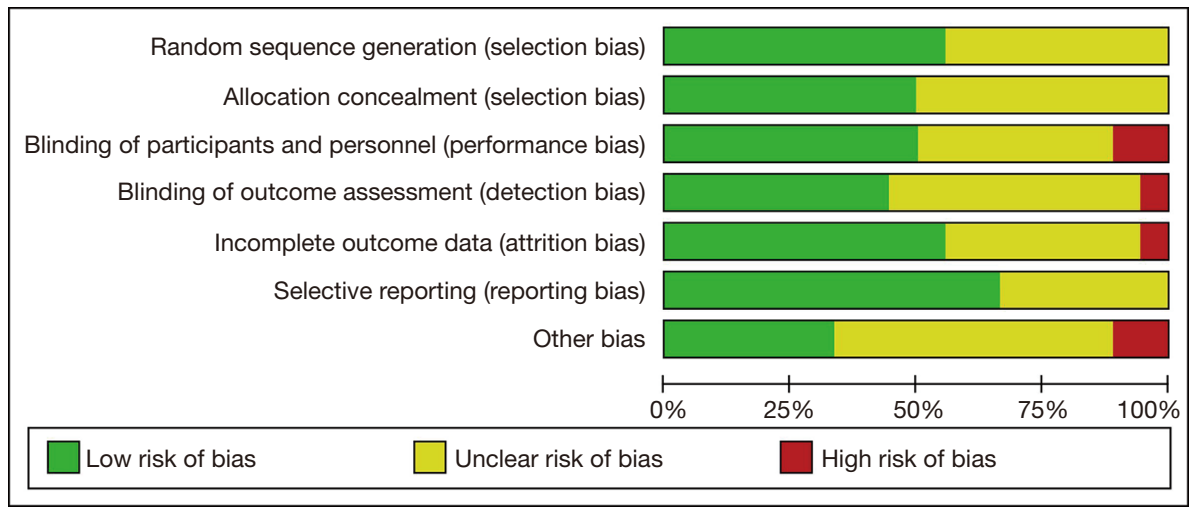

Figure 3 The risk of bias evaluation results.

was the most common (95\% CI: $0.79-0.97$ ), followed by thickening of the blood vessels (95\% CI: $0.63-0.78$ ), and pleural thickening (95\% CI: 0.02-0.15).

\section{Lesion distribution}

Figure 6 shows the meta-analysis results of lesion distribution. The heterogeneity test results found that external subpleural distribution, external semicenter distribution, double lung distribution, and single lung distribution all exhibited high heterogeneity $\left(\mathrm{I}^{2}=98.8 \%>50 \%, \mathrm{P}<0.01\right)$. Of the 18 studies, 12 reported that the lesions were externally subpleural (95\% CI: $0.132-$ $0.173)$, and 6 reported that the lesions were distributed in a single lobe (95\% CI: 0.598-0.841).

\section{Lesion morphology changes}

Figure 7 shows the results of the meta-analysis on lesion morphology changes. The heterogeneity test results showed that the morphology of the lesions was cord-like (95\% CI: 0.092-0.172), grid-like (95\% CI: 0.152-0.193), patchy (95\% CI: $0.192-0.313)$, and nodular (95\% CI: 0.591-0.745).

\section{Changed lesion density}

Figure 8 shows the results of the meta-analysis on the changed lesion density. The heterogeneity test results showed that the lesion density exhibited consolidation (95\% CI: 0.182-0.253), crazy-paving (95\% CI: 0.452-0.793), empty-bronchus sign (95\% CI: 0.292-0.415), and groundglass opacity (95\% CI: $0.521-0.765)$.

\section{Accompanying CT signs}

Figure 9 shows the meta-analysis results of accompanying CT signs. The heterogeneity test results showed that the accompanying CT signs included pleural effusion (95\% CI: 0.021-0.045) and pleural thickening (95\% CI: 0.252-0.453).

\section{Publication bias}

A funnel plot of ground-glass opacity (Figure 10) was generated by Rev Man 5.3. Meta-analysis was conducted on the ground glass change indicators of infectious pneumonia patients. The funnel plot showed that the circles in the included studies were distributed near the midline and basically symmetrical, indicating that there was no bias in publication and the conclusions obtained were reliable.

\section{Discussion}

The progress of COVID-19 has resulted in an increasing number of patients, and its diagnosis and treatment has attracted worldwide attention (18). In this study, 18 studies met the inclusion criteria, involving a total of 1,586 cases. The items proposed by the NICE were adopted for quality evaluation (19). There were 7 studies scoring 6-8 (38.89\%), 10 studies scoring 3-6 (55.55\%), and 1 scoring 0-2 (5.56\%), suggesting that the included literature had good reliability (20).

Early CT findings are usually multiple lesions in both lungs, which are often irregular, fan-shaped, or flaky or almost round, and generally do not involve the whole lung segment. The lesion density is not uniform, often showing small localized patches or large flake ground glass shadow. In the advanced stage, CT shows increased lesion 


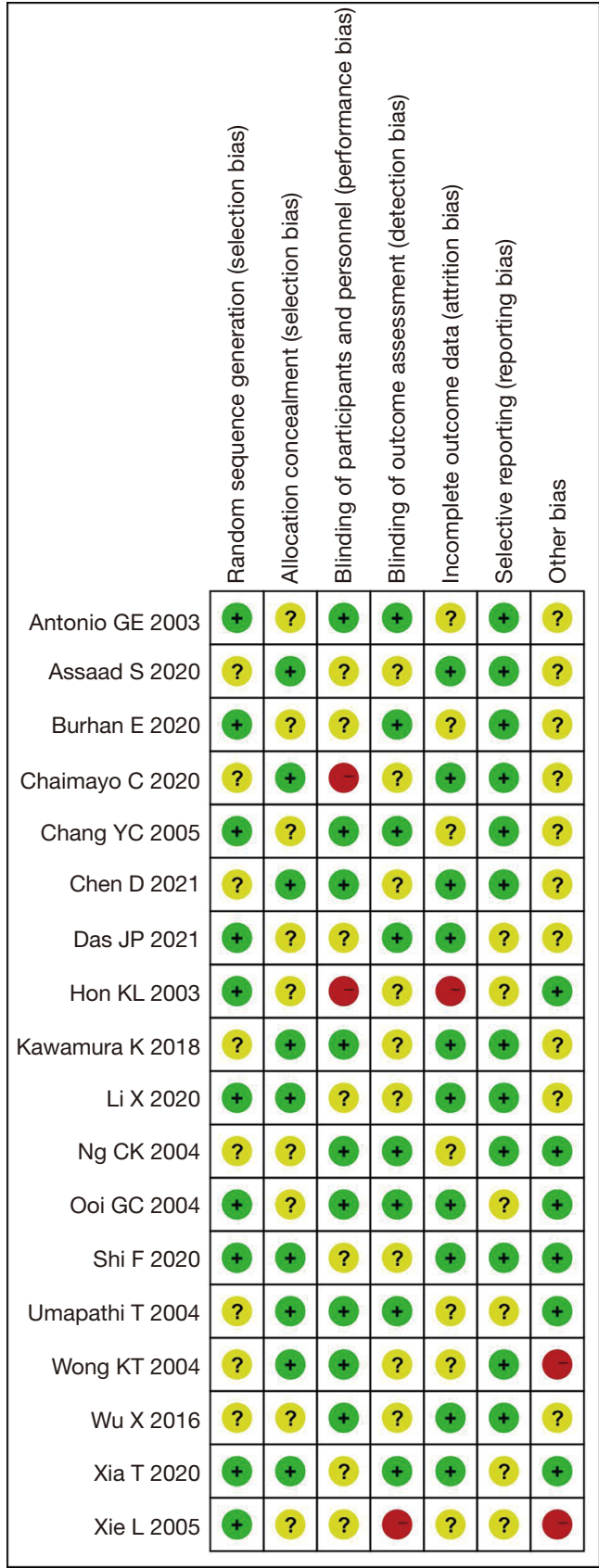

Figure 4 The risk of bias evaluation results. “+”: low risk; “_”: high risk; "?": unclear.

distribution area and increased lesion density, irregular, wedge-shaped or fan-shaped consolidation appeared in the lesions, with unclear boundaries, thickened bronchovascular bundles or multifocal lung consolidation under the pleura. In the critical phase, CT findings shows diffuse lesions in both lungs, with the appearance of "white lung" when most of the lungs are involved, and the diaphragmatic surface is elevated. The lesions are of uneven density, with air bronchial signs and bronchiectasis, and patchy ground glass shadows in areas of non-consolidation. The CT findings during the absorption period are density diminishes, lung consolidation disappears gradually, ground glass shadows are fully absorbed, and exudates are absorbed or organized by the body. The CT findings of bacterial pneumonia are mainly patchy consolidation shadows, which often accompanied by pneumonic bronchial signs; the CT findings of viral pneumonia are mainly multilobular peripheral ground glass shadows accompanied by lithotrite signs and interstitial thickening; and the CT findings of mycoplasma pneumonia are mainly interstitial inflammatory reactions, which distributed in double lower lungs, with thickening of bronchiole walls.

Of the 18 studies, the heterogeneity test results showed that among the CT signs, lung ground-glass shadow, lung consolidation shadow, and lung ground-glass shadow combined with consolidation exhibited high heterogeneity, with $\mathrm{I}^{2}=96.4 \%>50 \%$. Ground-glass shadow was the most common (95\% CI: 0.79-0.97), followed by thickening of the blood vessels (95\% CI: 0.63-0.78), and pleural thickening (95\% CI: 0.02-0.15). Asadollahi-Amin et al. [2020] (21) pointed out that diagnosis based on lung imaging is accurate. The CT imaging characteristics change with the disease condition, suggesting that $\mathrm{CT}$ images can be used for real-time diagnosis and evaluation (22).

Of the 18 studies, 12 reported that the lesions were externally subpleural (95\% CI: 0.132-0.173), and 6 reported that the lesions were distributed in a single lobe $(95 \% \mathrm{CI}$ : $0.598-0.841)$. The heterogeneity test results showed that the morphology of the lesions was cord-like (95\% CI: 0.092-0.172), grid-like (95\% CI: 0.152-0.193), patchy (95\% CI: 0.192-0.313), and nodular (95\% CI: 0.591-0.745). Furthermore, ground-glass opacity was a typical CT manifestation of patients with infectious pneumonia (23). Therefore, it is highly necessary to combine CT characteristics to diagnose and treat infectious pneumonia.

The heterogeneity test results showed that the accompanying CT signs included pleural effusion (95\% CI: $0.021-0.045)$ and pleural thickening (95\% CI: 0.252-0.453). Some patients also had lymph node enlargement. If there is pleural effusion combined with lymph node enlargement, it indicates that the patient may have an adverse reaction and the condition will become worse.

Patients with common type showed no obvious lesions on lung CT after healing, while patients with severe type showed 


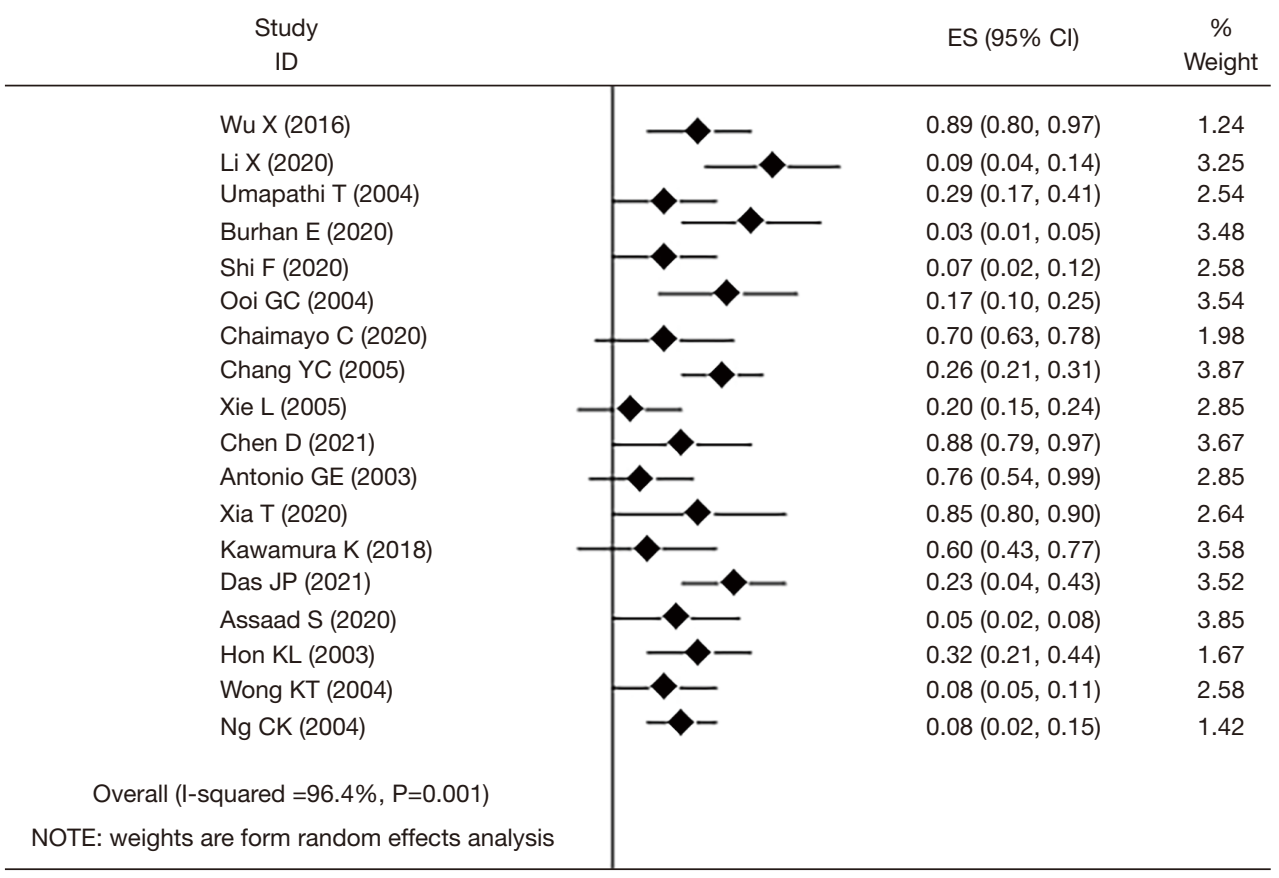

Figure 5 Forest plot showing the CT signs of infectious pneumonia. CT, computed tomography; ES, effect size; CI, confidence interval.

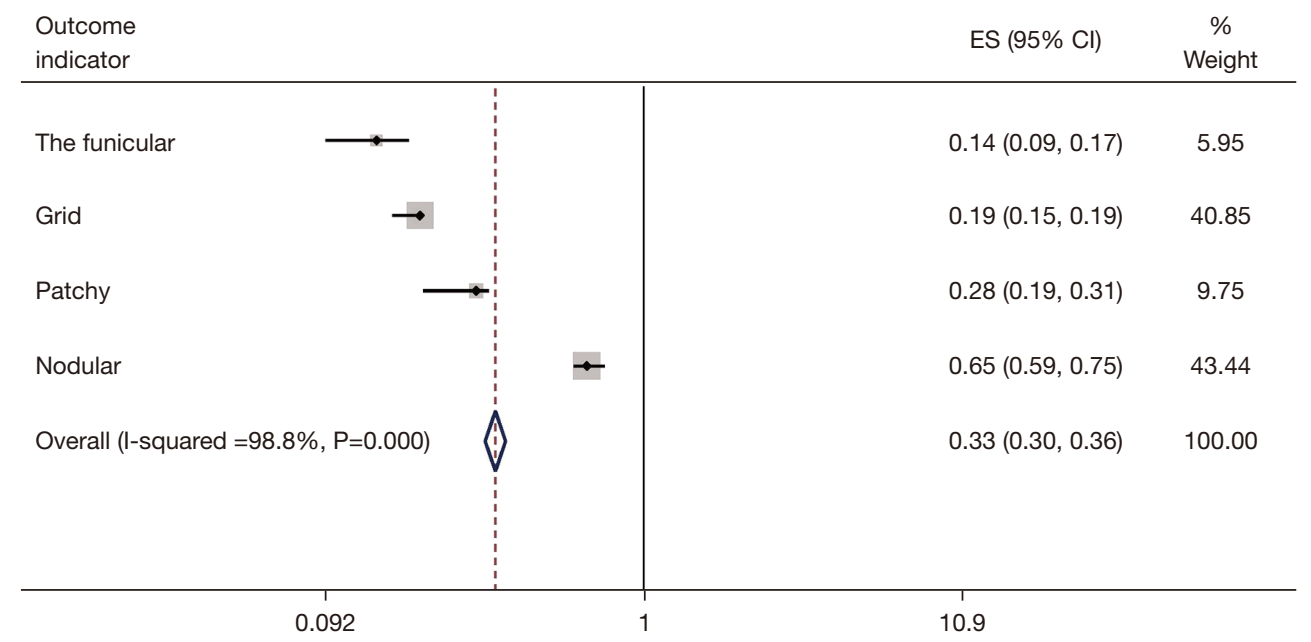

Figure 6 Meta-analysis of lesion distribution. ES, effect size; CI, confidence interval.

different degrees of lesion retention on lung CT, which was consistent with the results of Chen et al. [2020] (24). Of the 18 RCTs included in this study, 5 had the correct random allocation method, accounting for $27.78 \%$, and only 2 had detailed allocation concealment, accounting for $16.67 \%$. One study was evaluated using the blinding method, accounting for $5.55 \%$, which was not adopted in the other studies. Meta-analysis was conducted on the ground glass change indicators of infectious pneumonia patients. The funnel plot showed that the circles in the included studies were distributed near the midline and basically symmetrical, indicating that there was no bias in publication and the conclusions obtained were reliable. In the prevention of infectious diseases, laws and regulations should be taken as the guidance, corresponding measures should be taken in time according to the progress of the 


\begin{tabular}{|c|c|c|c|}
\hline Outcome indicator & & ES $(95 \% \mathrm{Cl})$ & $\begin{array}{c}\% \\
\text { Weight }\end{array}$ \\
\hline Single lung involvement $\longrightarrow$ & & $0.12(0.08,0.15)$ & 11.32 \\
\hline One lung is involved & & $0.16(0.13,0.17)$ & 54.43 \\
\hline Multiple lobes are involved & $\rightarrow$ & $0.75(0.60,0.84)$ & 34.25 \\
\hline Overall (I-squared $=99.1 \%, P=0.000)$ & & $0.26(0.24,0.29)$ & 100.00 \\
\hline 0.084 & & 11.9 & \\
\hline
\end{tabular}

Figure 7 Meta-analysis on lesion morphology changes. ES, effect size; CI, confidence interval.

\begin{tabular}{|c|c|c|}
\hline Outcome indicator & ES (95\% Cl) & $\begin{array}{c}\% \\
\text { Weight }\end{array}$ \\
\hline Consolidation & $0.24(0.18,0.25)$ & 33.82 \\
\hline Stone road & $0.59(0.45,0.79)$ & 11.61 \\
\hline Air bronchial sign & $0.38(0.29,0.41)$ & 29.70 \\
\hline Ground glass sample & $0.65(0.52,0.76)$ & 24.87 \\
\hline Overall (I-squared $=95.8 \%, P=0.000)$ & $0.39(0.35,0.43)$ & 100.00 \\
\hline
\end{tabular}

Figure 8 Meta-analysis on the changed lesion density. ES, effect size; CI, confidence interval.

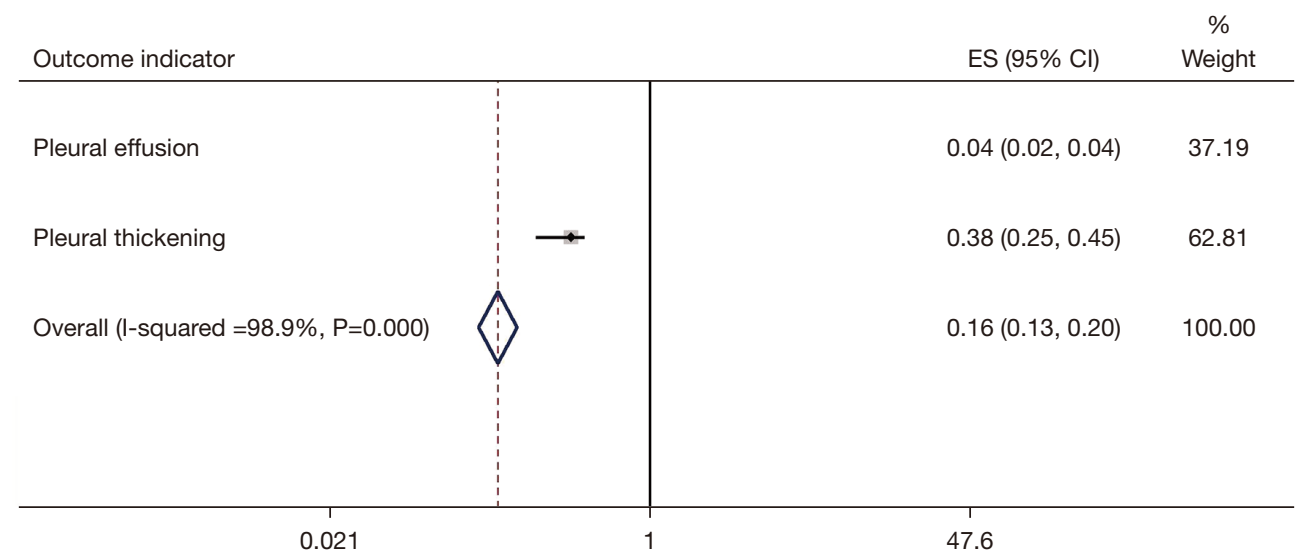

Figure 9 Meta-analysis results of accompanying CT signs. CT, computed tomography; ES, effect size; CI, confidence interval. 


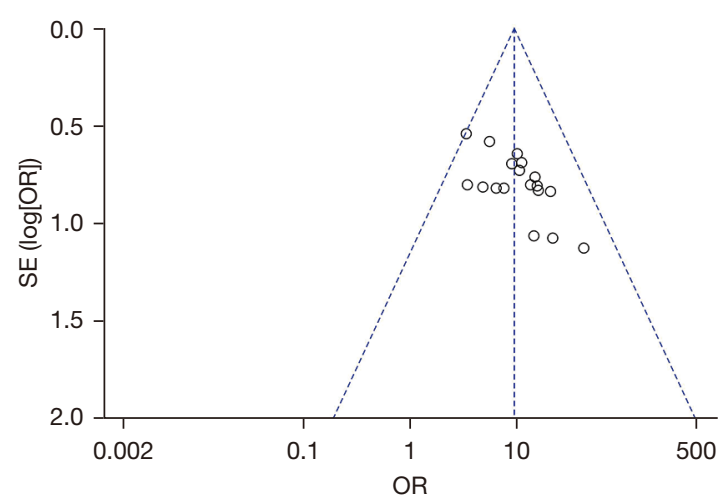

Figure 10 Funnel plots of ground-glass opacity included in the literature. SE, standard error; OR, odds ratio.

epidemic, the publicity function of social media should be given full play, information should be kept unblocked, and public opinion should be guided. In an emergency outbreak, public health authorities need to continuously improve their management capacity and efficiency, and make the right response measures in the first time.

\section{Conclusions}

In this meta-analysis, RCTs involving patients with infectious pneumonia were selected to evaluate the diagnostic value of CT imaging. It was found that groundglass opacity was a typical CT manifestation in patients with infectious pneumonia, and some patients also showed thickening of blood vessels. It was concluded that CT signs were instrumental in the diagnosis, evaluation, and prognosis of infectious pneumonia. However, some limitations should be noted. The classification of patients with infectious pneumonia is not accurate enough. The subjects may also have hypertension and cardiovascular and cerebrovascular diseases, among others, which are not taken into consideration, reducing the power of the study. The included subjects were from different countries and regions, and the analysis results may be affected by the subjective factors of the equipment and doctors. In conclusion, CT is instrumental in diagnosing infectious pneumonia, and corresponding treatment plans can be determined based on CT manifestations.

\section{Acknowledgments}

Funding: This study was supported by Premium Open Online Courses of Pingdingshan University (jpzx18108).

\section{Footnote}

Reporting Checklist: The authors have completed the PRISMA reporting checklist. Available at https://dx.doi. org/10.21037/apm-21-2101

Conflicts of Interest: All authors have completed the ICMJE uniform disclosure form (available at https://dx.doi. org/10.21037/apm-21-2101). The authors have no conflicts of interest to declare.

Ethical Statement: The authors are accountable for all aspects of the work in ensuring that questions related to the accuracy or integrity of any part of the work are appropriately investigated and resolved.

Open Access Statement: This is an Open Access article distributed in accordance with the Creative Commons Attribution-NonCommercial-NoDerivs 4.0 International License (CC BY-NC-ND 4.0), which permits the noncommercial replication and distribution of the article with the strict proviso that no changes or edits are made and the original work is properly cited (including links to both the formal publication through the relevant DOI and the license). See: https://creativecommons.org/licenses/by-nc-nd/4.0/.

\section{References}

1. Wu X, Dong D, Ma D. Thin-section computed tomography manifestations during convalescence and longterm follow-up of patients with severe acute respiratory syndrome (SARS). Med Sci Monit 2016;22:2793-9.

2. Li X, Zeng W, Li X, et al. CT imaging changes of corona virus disease 2019 (COVID-19): a multi-center study in Southwest China. J Transl Med 2020;18:154.

3. Umapathi T, Kor AC, Venketasubramanian N, et al. Large artery ischaemic stroke in severe acute respiratory syndrome (SARS). J Neurol 2004;251:1227-31.

4. Burhan E, Prasenohadi P, Rogayah R, et al. Clinical progression of COVID-19 patient with extended incubation period, delayed RT-PCR time-to-positivity, and potential role of chest CT-scan. Acta Med Indones 2020;52:80-3.

5. Shi F, Yu Q, Huang W, et al. 2019 novel coronavirus (COVID-19) pneumonia with hemoptysis as the initial symptom: CT and clinical features. Korean J Radiol 2020;21:537-40.

6. Ooi GC, Khong PL, Müller NL, et al. Severe acute 
respiratory syndrome: temporal lung changes at thinsection CT in 30 patients. Radiology 2004;230:836-44.

7. Chaimayo C, Kaewnaphan B, Tanlieng N, et al. Rapid SARS-CoV-2 antigen detection assay in comparison with real-time RT-PCR assay for laboratory diagnosis of COVID-19 in Thailand. Virol J 2020;17:177.

8. Chang YC, Yu CJ, Chang SC, et al. Pulmonary sequelae in convalescent patients after severe acute respiratory syndrome: evaluation with thin-section CT. Radiology 2005;236:1067-75.

9. Xie L, Liu Y, Xiao Y, et al. Follow-up study on pulmonary function and lung radiographic changes in rehabilitating severe acute respiratory syndrome patients after discharge. Chest 2005;127:2119-24.

10. Chen D, Jiang X, Hong Y, et al. Can chest CT features distinguish patients with negative from those with positive initial RT-PCR results for coronavirus disease (COVID-19)? AJR Am J Roentgenol 2021;216:66-70.

11. Antonio GE, Wong KT, Hui DS, et al. Thin-section CT in patients with severe acute respiratory syndrome following hospital discharge: preliminary experience. Radiology 2003;228:810-5.

12. Xia T, Li J, Gao J, et al. Small solitary ground-glass nodule on CT as an initial manifestation of coronavirus disease 2019 (COVID-19) pneumonia. Korean J Radiol 2020;21:545-9.

13. Kawamura K, Ichikado K, Takaki M, et al. Adjunctive therapy with azithromycin for moderate and severe acute respiratory distress syndrome: a retrospective, propensity score-matching analysis of prospectively collected data at a single center. Int J Antimicrob Agents 2018;51:918-24.

14. Das JP, Yeh R, Schöder H. Clinical utility of perfusion (Q)single-photon emission computed tomography (SPECT)/ CT for diagnosing pulmonary embolus (PE) in COVID-19 patients with a moderate to high pre-test probability of PE. Eur J Nucl Med Mol Imaging 2021;48:794-9.

15. Assaad S, Avrillon V, Fournier ML, et al. High mortality rate in cancer patients with symptoms of COVID-19 with or without detectable SARS-COV-2 on RT-PCR. Eur J

Cite this article as: Liu Y, Zhou X, Liu X, Yi Y, Zhang S, Wang J, Yuan F. Systematic review and meta-analysis of the CT imaging characteristics of infectious pneumonia. Ann Palliat Med 2021;10(10):10414-10424. doi: 10.21037/apm-21-2101
Cancer 2020;135:251-9.

16. Hon KL, Leung CW, Cheng WT, et al. Clinical presentations and outcome of severe acute respiratory syndrome in children. Lancet 2003;361:1701-3.

17. Wong KT, Antonio GE, Hui DS, et al. Severe acute respiratory syndrome: thin-section computed tomography features, temporal changes, and clinicoradiologic correlation during the convalescent period. J Comput Assist Tomogr 2004;28:790-5.

18. Ng CK, Chan JW, Kwan TL, et al. Six month radiological and physiological outcomes in severe acute respiratory syndrome (SARS) survivors. Thorax 2004;59:889-91.

19. Shang Y, Xu C, Jiang F, et al. Clinical characteristics and changes of chest CT features in 307 patients with common COVID-19 pneumonia infected SARS-CoV-2: a multicenter study in Jiangsu, China. Int J Infect Dis 2020;96:157-62.

20. Meng H, Xiong R, He R, et al. CT imaging and clinical course of asymptomatic cases with COVID-19 pneumonia at admission in Wuhan, China. J Infect 2020;81:e33-9.

21. Asadollahi-Amin A, Hasibi M, Ghadimi F, et al. Lung involvement found on chest CT scan in a pre-symptomatic person with SARS-CoV-2 infection: a case report. Trop Med Infect Dis 2020;5:56.

22. Varble N, Blain M, Kassin M, et al. CT and clinical assessment in asymptomatic and pre-symptomatic patients with early SARS-CoV-2 in outbreak settings. Eur Radiol 2021;31:3165-76.

23. Pautrat K, Chergui N. SARS-CoV-2 infection may result in appendicular syndrome: chest CT scan before appendectomy. J Visc Surg 2020;157:S63-4.

24. Chen X, Zhang G, Hao SY, et al. Similarities and differences of early pulmonary CT features of pneumonia caused by SARS-CoV-2, SARS-CoV and MERS-CoV: comparison based on a systemic review. Chin Med Sci J 2020;35:254-61.

(English Language Editor: C. Betlazar-Maseh) 